\title{
Fatores impeditivos da realização do exame Papanicolau em idosas: uma revisão integrativa
}

\author{
Impeditive factors in carrying out the Papanicolaou test in elderly: an integrative review
}

Factores que impiden la realización del Papanicolaou en ancianas: una revisión integradora

Gabrielly Freitas da Silva ${ }^{1 *}$, Josilene Vieira Ramos ${ }^{1}$, Larissa Simões Moreira Batista ${ }^{1}$, Raquel de Souza Queiroz ${ }^{1}$, Danielle Mendonça Jatobá ${ }^{1}$, Luiza Jatobá de Hollanda ${ }^{2}$, Ana Clara Veloso de Assis ${ }^{1}$, Brenda Tayrine Tavares Souza ${ }^{1}$, Albênica Paulino dos Santos Bontempo'.

\section{RESUMO}

Objetivo: Identificar os fatores impeditivos para a realização do exame Papanicolau em idosas. Métodos: Trata-se de uma revisão integrativa da literatura realizada nas bases de dados online da Biblioteca Virtual em Saúde como Literatura Latino-Americana e do Caribe em Ciências da Saúde (LILACS), Bancos de Dados em Enfermagem (BDENF), Biblioteca Eletrônica Científica Online (SciELO) e Sistema Online de Busca e Análise de Literatura Médica (MEDLINE). Foram utilizados artigos em português, publicados nos últimos 5 anos e que continham os descritores "Exame Papanicolau", "Pessoa idosa", "Cuidado preventivo" e "Saúde da mulher". Resultados: Inicialmente foram encontrados 103 artigos e destes foram selecionados 8 que identificaram os principais fatores que influenciam a não realização do exame Papanicolau em idosas. Considerações finais: O enfermeiro é o profissional responsável pela coleta do material citológico vaginal durante a consulta de enfermagem e cabe a ele identificar fatores impeditivos, buscando trabalhar com ações educativas no rompimento de barreiras que impedem a adesão das idosas ao exame preventivo.

Palavras-chave: Exame Papanicolau, Pessoa idosa, Cuidado preventivo, Saúde da mulher.

\begin{abstract}
Objective: To identify the factors that may prevent the Papanicolaou test from being performed in elderly women. Methods: This is an integrative literature review, made through the online databases of the Virtual Health Library (VHL) such as the Latin American \& Caribbean Health Sciences Literature (LILACS), Databases in Nursing (BDENF), Scientific Electronic Library Online (SciELO) and the Medical Literature Analysis and Retrieval System Online (MEDLINE). Inclusion criteria were articles published in Portuguese and in the past five years, with the following descriptors: Pap smear; Elderly people; Preventive care; Women's health. Results: 103 articles were found, from which 8 were selected for identifying the main factors that prevent the smear test from being performed on elderly women. Final considerations: The nurse is responsible for collecting the vaginal cytological material in the office during a nursing consultation and it is up to them to identify impeding factors, seeking to work with educational actions to break down barriers that prevent elderly women's adherence to the Preventive Exam.
\end{abstract}

Keywords: Papanicolaou test, Aged, Preventive medicine, Women's health.

\section{RESUMEN}

Objetivo: Identificar los factores que obstaculizan la realización del Papanicolaou en ancianas. Métodos: Se trata de una revisión integrativa de la literatura realizada en las bases de datos en línea de la Biblioteca Virtual en Salud como Literatura Latinoamericana y del Caribe en Ciencias de la Salud (LILACS), Bases de Datos de Enfermería (BDENF), Biblioteca Científica Electrónica en Línea (SciELO) y Análisis y Recuperación de Literatura Médica Sistema en Línea (MEDLINE). Se utilizaron artículos en portugués, publicados en los últimos 5 años y que contenían los descriptores "Papanicolau", "Anciano", "Cuidados preventivos" y "Salud de la mujer". Resultados: Inicialmente fueron encontrados 103 artículos y de estos fueron seleccionados 8, los cuales identificaron los principales factores que influyen en la no realización del Papanicolaou en ancianas. Consideraciones finales: El enfermero es el profesional responsable por la recolección del material

${ }^{1}$ Centro Universitário do Distrito Federal (UDF), Brasília - DF. *E-mail: gabriellyfreitas21.02@gmail.com

2 Universidade de Brasília (UnB), Brasília - DF. 
citológico vaginal durante la consulta de enfermería y le corresponde a él identificar los factores obstaculizadores, buscando trabajar con acciones educativas para romper las barreras que impiden a las ancianas adherirse al examen preventivo.

Palabras clave: Prueba de Papanicolaou, Anciano, Medicina preventiva, Salud de la mujer.

\section{INTRODUÇÃO}

O Câncer de Colo de Útero (CCU) é uma neoplasia maligna que pode ser prevenida através do exame Papanicolau, recomendado para mulheres com idade entre 25 e 64 anos ou que já possuam atividade sexual, antes mesmo dos 25 anos (MOREIRA AS e ANDRADE EGS, 2018). O principal objetivo do rastreamento é detectar o CCU em sua fase pré-clínica para que o tratamento possa ser efetivo e possibilitar a cura e a diminuição da morbidade (CAMPOS EA, et al., 2017). Embora esse exame seja a forma mais eficaz de prevenção do $\mathrm{CCU}$, a aceitação e os hábitos preventivos por parte das mulheres ainda apresentam barreiras devido aos padrões e valorização dos aspectos culturais que dificultam mudanças comportamentais (CAMPOS EA, et al., 2017).

No Brasil, a maior incidência de casos de CCU ocorre nas regiões Norte, com 26,24 casos a cada 100 mil mulheres, na região Nordeste, com 16,10 casos a cada 100 mil mulheres, e na região Centro-Oeste, com 12,35 casos a cada 100 mil mulheres (INSTITUTO NACIONAL DE CÂNCER (INCA), 2019). Estudo realizado na cidade de Bebedouro, no estado de São Paulo, sobre a aderência ao exame preventivo, foi relatado que $38 \%$ das mulheres fazem o exame anualmente, $39 \%$ estão com o exame preventivo atrasado e $23 \%$ nunca realizaram este exame, sendo os fatores para a não realização relacionados a hábitos culturais e ao medo (OLIVEIRA DS, et al., 2019).

O Exame Ginecológico Papanicolau (EGP) teve sua origem no início do século XX com o Dr. Geórgios Papanicolau, médico grego considerado o pai da citopatologia, é um método utilizado nas Unidades Básicas de Saúde (UBS) para detectar lesões no colo do útero (DIAS EG, et al., 2017). Espera-se que o rastreamento do CCU alcance mais de $80 \%$ das mulheres, de acordo com as recomendações da Organização Mundial de Saúde (OMS). A coleta do material cervical e endocervical acontece através da raspagem nas regiões do fundo do útero e é considerado um método simples e de fácil realização (PINHEIRO HDM, et al., 2018).

O envelhecimento da população tem acelerado rapidamente devido a diminuição da fecundidade e da mortalidade, o que gera uma maior expectativa de vida, fator que vem acompanhado de doenças crônicas não transmissíveis, como as neoplasias. Diante dessas modificações populacionais, os idosos buscam uma velhice ativa, inclusive, com a vivência da sexualidade. Entretanto, algumas idosas desconsideram o sexo seguro e não fazem uso do preservativo em virtude da infertilidade. Cabe ao profissional da saúde identificar tal comportamento que é considerado de risco para o desenvolvimento de neoplasias (SARDINHA AH, et al., 2021; SANTOS AD, et al., 2019).

Considerando o envelhecimento populacional brasileiro, estima-se que em 2050 haverá um aumento na população das mulheres idosas, que passará de 18\% para cerca de 30,8\% (BARRETO AMMA, et al., 2018). O estudo de Acosta DF, et al. (2017), aponta que as mulheres idosas apresentam maior resistência à realização do exame preventivo, fato que desencadeia preocupações a nível de saúde pública devido às complicações severas do CCU, que podem até mesmo levar à morte.

Diante do exposto, o presente estudo teve por objetivo identificar os possíveis fatores impeditivos da realização do EGP em mulheres idosas.

\section{MÉTODOS}

Trata-se de uma revisão integrativa realizada através da plataforma Biblioteca Virtual em Saúde (BVS) utilizando as bases de dados Literatura Latino-Americana e do Caribe em Ciências da Saúde (LILACS), Bancos de Dados em Enfermagem (BDENF), Biblioteca Eletrônica Científica Online (SciELO), Sistema Online de Busca e Análise de Literatura Médica (MEDLINE). Buscas adicionais foram realizadas no Google Acadêmico e também foram utilizados Manuais do Ministério da Saúde (MS) e do Instituto Nacional do Câncer 
(INCA). Foram utilizados os seguintes Descritores: "Exame Papanicolau", "Pessoa Idosa", "Cuidado preventivo" e "Saúde da mulher", juntamente com o operador booleano "AND".

A pesquisa foi realizada utilizando como critérios de inclusão artigos publicados em português, nos últimos 5 anos e com a temática que atendesse ao objetivo. Foram excluídos artigos duplicados, cartas aos editores, relatos de casos e os que não atenderam aos critérios.

No processo de seleção foram encontrados 103 artigos, onde 95 foram eliminados pelos critérios de exclusão, restando 8 artigos que atendiam ao objetivo da pesquisa. Para análise dos dados foram seguidas as orientações do Relatório Preferido para Revisões Sistemáticas e Meta-Análises (PRISMA). A seleção dos artigos teve três etapas: leitura dos títulos, leitura dos resumos e leitura na íntegra. Por fim, foram extraídos os resultados e conclusões mais relevantes dos estudos para compilação dos achados.

\section{RESULTADOS}

Foram localizados 103 artigos usando a metodologia empregada. Destes, foram removidos 37 artigos duplicados e 58 que não atendiam aos critérios de elegibilidade. Foram selecionados 8 artigos após leitura detalhada. Os detalhes da busca estão esquematizados no fluxograma da Figura 1.

Figura 1 - Diagrama de Fluxo PRISMA.

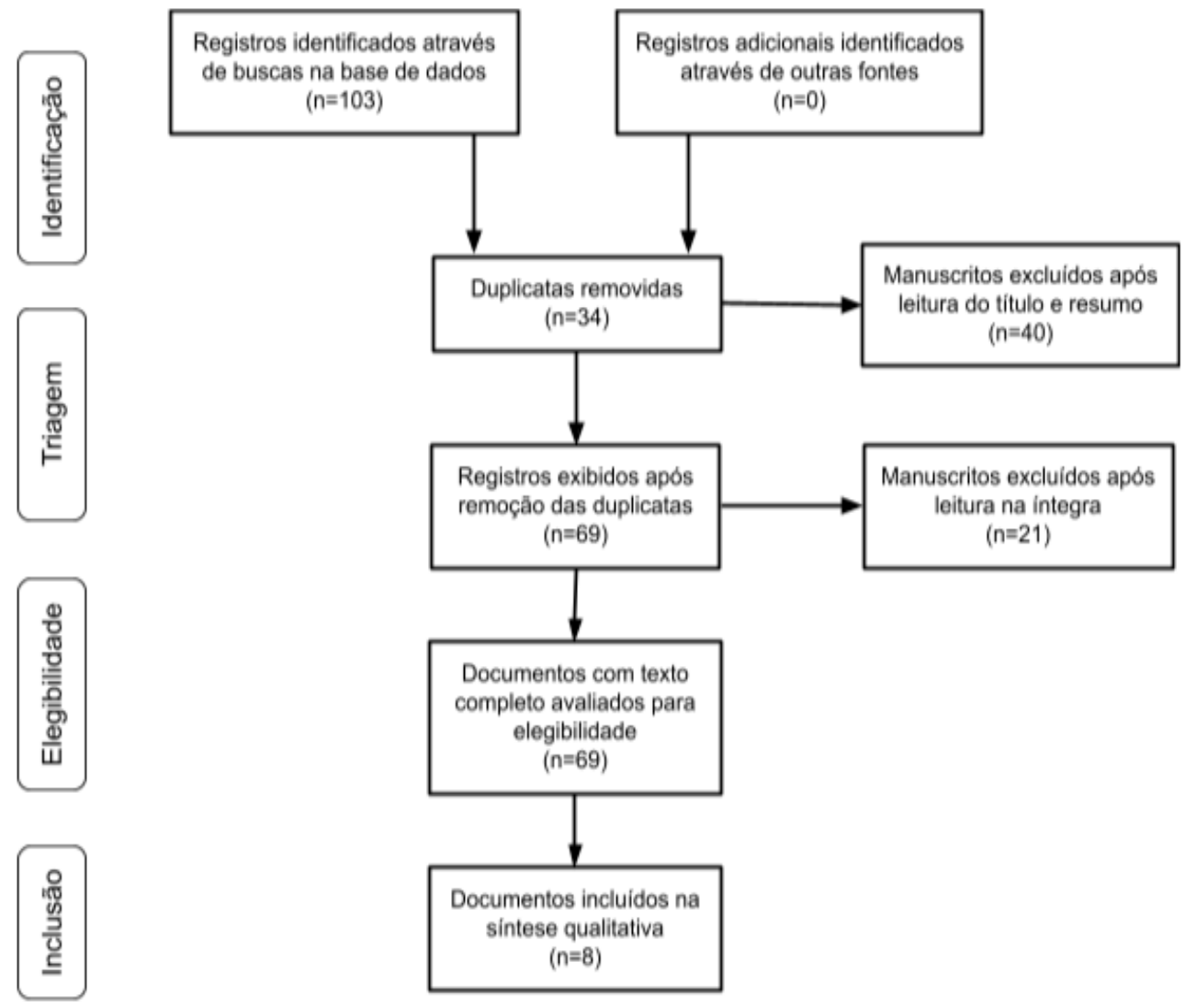

Fonte: Silva GF, et al., 2022.

O Quadro 1 traz uma síntese dos principais achados nos estudos selecionados para este artigo, destacando trechos relacionados aos fatores impeditivos de realização do exame Papanicolau em mulheres idosas. 
Quadro 1 - Síntese dos principais achados relacionados aos fatores impeditivos de realização do exame Papanicolau em mulheres idosas.

\begin{tabular}{|c|c|c|}
\hline $\mathbf{N}$ & Autores (Ano) & $\begin{array}{l}\text { Principais achados } \\
\end{array}$ \\
\hline 1 & $\begin{array}{l}\text { FEITOSA LMH, et al. } \\
(2017) .\end{array}$ & $\begin{array}{l}\text { Estudo descritivo. Analisar o conhecimento, as atitudes que devem ser } \\
\text { tomadas e a prática das mulheres idosas frente ao exame colpocitológico; } \\
\text { as idosas apresentaram conhecimento insuficiente sobre o colpocitológico } \\
\text { mas, a prática e atitude não foram afetadas pois costumam realizá-lo } \\
\text { quando estão saudáveis. }\end{array}$ \\
\hline 2 & $\begin{array}{c}\text { BATISTA AFC e } \\
\text { CALDAS CP (2017). }\end{array}$ & $\begin{array}{l}\text { Revisão abrangente da literatura. Discutir os motivos que influenciam a } \\
\text { adesão de idosas aos programas de prevenção do CCU; os fatores que } \\
\text { impedem a adesão do exame são a dificuldade de acesso ao serviço de } \\
\text { saúde, preconceito dos próprios idosos em relação à velhice, falta de } \\
\text { capacitação dos profissionais, pouco investimento em educação em saúde. }\end{array}$ \\
\hline 3 & $\begin{array}{c}\text { ACOSTA DF, et al. } \\
(2017)\end{array}$ & $\begin{array}{l}\text { Estudo exploratório, descritivo, de abordagem qualitativa. Observar o } \\
\text { entendimento das idosas de uma unidade de ESF em relação ao EGP; o } \\
\text { desconhecimento da importância da sua realização, a vergonha, o medo } \\
\text { de sentir dor durante a coleta do exame e o medo do diagnóstico são } \\
\text { fatores que impedem que as idosas busquem realizar o exame } \\
\text { papanicolau. }\end{array}$ \\
\hline 4 & $\begin{array}{l}\text { TAVARES DL, et al. } \\
(2018) .\end{array}$ & $\begin{array}{l}\text { Estudo descritivo. Avaliar idosas que realizaram o EGP cérvico-vaginal e } \\
\text { microflora no município de Santa Maria, RS; a falta de atividade sexual, o } \\
\text { medo e a vergonha levam idosas a não realizarem o exame citopatológico } \\
\text { no período de } 2010 \text { a } 2013 \text {. }\end{array}$ \\
\hline 5 & SILVA JP, et al. (2018). & $\begin{array}{l}\text { Estudo descritivo e analítico, com abordagem quantitativa. Caracterizar os } \\
\text { fatores que impedem as mulheres entre } 40 \text { e } 65 \text { anos de idade a não } \\
\text { realizarem o EGP; a maioria das mulheres entrevistadas costumam realizar } \\
\text { o EGP todos os anos, e às vezes não o fazem por vergonha e medo dos } \\
\text { resultados do exame. }\end{array}$ \\
\hline 6 & LEITE BO, et al. (2019). & $\begin{array}{l}\text { Estudo descritivo com abordagem qualitativa. Descrever a percepção das } \\
\text { idosas sobre o EGP; muitas idosas têm opiniões contrárias sobre o EGP, } \\
\text { demonstrando ansiedade, tensão, vergonha e medo de fazer o exame. }\end{array}$ \\
\hline 7 & $\begin{array}{l}\text { OLIVEIRA DS, et al. } \\
(2019) .\end{array}$ & $\begin{array}{l}\text { Estudo de revisão integrativa da literatura. Discutir a atuação da enfermeira } \\
\text { frente aos fatores que interferem na adesão das idosas ao EGP; A equipe } \\
\text { de enfermagem tem um papel fundamental na prevenção do CCU } \\
\text { realizando educação em saúde, busca ativa e principalmente um } \\
\text { atendimento humanizado, visto que, fatores como: vergonha, medo, } \\
\text { desconhecimento sobre a importância do exame impedem a realização do } \\
\text { exame. }\end{array}$ \\
\hline 8 & $\begin{array}{c}\text { SILVA MO, et al. } \\
\text { (2020). }\end{array}$ & $\begin{array}{l}\text { Revisão sistemática da literatura com metassíntese. Investigar na literatura } \\
\text { os fatores relacionados a não adesão ao EGP em idosas; o } \\
\text { desconhecimento da importância do EGP, receio de expor a genitália, } \\
\text { principalmente aos profissionais do sexo masculino são fatores } \\
\text { relacionados a não adesão. }\end{array}$ \\
\hline
\end{tabular}

Legenda: EGP: Exame Ginecológico Papanicolau; CCU: Câncer de Colo de Útero: ESF: Estratégia Saúde Família. Fonte: SILVA GF, et al., 2022.

\section{DISCUSSÃO}

O EGP é uma ferramenta capaz de detectar células cancerígenas e é considerado o exame "padrão ouro" pelo Ministério da Saúde (MS) (MINISTÉRIO DA SAÚDE, 2019). Este exame pode ser realizado nas unidades de saúde pública ou privada que possuam profissionais habilitados e tem como foco mulheres com a vida sexual ativa (SILVA JP, et al.,2018). O MS preconiza mulheres com faixa etária de 25 a 64 anos por terem uma maior existência de lesões de alto grau capazes de serem tratadas de forma efetiva e não evoluindo para o câncer (INCA, 2021). É primordial a orientação sobre a importância do EGP, pois é o método principal para a detecção de lesões cervicais e sua execução regular permite que o diagnóstico seja precoce, impactando na redução da mortalidade por CCU (SIMÕES CS et al., 2021). 
O EGP se inicia com a introdução do espéculo no canal vaginal para observar as paredes do colo uterino e verificar se há presença de lesões visíveis e, em seguida, é coletada uma amostra tecidual de células localizadas na ectocérvice (parte externa do colo) e endocérvice (parte interna do colo), para que seja feita uma análise laboratorial (LIMA MB, et al., 2018; TAVARES DL, et al., 2018). Mulheres que apresentam dois resultados negativos anuais ficam dispensadas da realização do exame durante 3 anos, devido a lenta evolução do CCU(INCA, 2021).

Os fatores impeditivos para a não realização do EGP são a falta de conhecimento em relação ao exame, sentimentos como medo e desconforto, receio de sentir dor na realização do exame, constrangimento e vergonha da exposição da genitália ao profissional, principalmente do sexo masculino, e as influências dos saberes sociais e culturais (ANDRADE MI, et al., 2021; BATISTA AFC e CALDAS CP, 2017; FEITOSA LMH, et al., 2017). A não adesão também está relacionada à falta de capacitação dos profissionais, o distanciamento da idade fértil e ao pouco investimento na educação em saúde, fatores que contribuem para a vulnerabilidade das idosas e para maior prevalência de CCU (SILVA CM, et al., 2016; SILVA MO, et al., 2020; LIMA WF, et al., 2020; BAIA EM, et al., 2018; INCA, 2020; LEITE BO, et al., 2019).

O envelhecimento representa o desenvolvimento de modificações fisiológicas da vida e podem ser percebidas mudanças distintas em relação ao gênero (SILVA, et al., 2020). Tendo em vista os padrões femininos, estes são divididos pelas perdas e alterações corporais, onde menarca (primeira menstruação) e a menopausa (último ciclo menstrual) são fases fisiológicas que marcam a vida reprodutiva da mulher, porém, a prevenção de doenças dos órgãos genitais devem continuar mesmo após a menopausa (SILVA MO, et al., 2020; SELBAC MT, et al., 2018).

Para Oliveira DS, et al. (2019), a chegada da menopausa e o fim da idade fértil geram preconceitos, sendo um presságio para o afastamento das práticas preventivas e ginecológicas. Mulheres na menopausa tendem a não comparecer à realização do EGP e consultas ginecológicas, tornando-as mais suscetíveis ao desenvolvimento do CCU (OLIVEIRA DS, et al., 2019). É importante que o profissional de saúde compreenda os fatores culturais para que conheça o seu público-alvo (CARNEIRO C, et al., 2020).

Observa-se nos estudos que a realização do EGP faz emergir sentimentos como medo, vergonha e desconforto, e esses sentimentos são ainda mais significativos quando sua realização é com um profissional de saúde do sexo masculino, o que desencadeia um sentimento de intimidade violada o que, para as idosas, as deixam retraídas tornando difícil a realização do exame e do prosseguimento da assistência (OLIVEIRA DS, et al., 2019). A resistência ao EGP está relacionada à questão cultural e até mesmo à não permissão do parceiro para realização do exame que, como consequência, gera receio e sentimento de vergonha (IGLESIAS G, et al., 2019).

A exposição da genitália ao profissional gera constrangimento e vergonha e muitas idosas relatam que a exposição da sua genitália gera um bloqueio, o que causa um incômodo emocional e outros sentimentos desfavoráveis (SILVA MO, et al., 2020). O medo em relação à realização do EGP se dá por meio de experiências ruins de pessoas próximas e da própria experiência em coletas já realizadas, além dos pensamentos provenientes da chance de um resultado de CCU positivado, sendo necessário a orientação sobre o diagnóstico precoce e sua importância (SILVA JP, et al., 2018).

Baia EM, et al. (2018), salientam que as mulheres se sentem inferiores por terem sua genitália exposta e manuseada por um profissional, expondo que ainda não veem o EGP como algo comum e que a posição para a realização do exame gera sensação de incapacidade, não tendo o domínio sobre o próprio corpo, fato que gera tensão na hora do procedimento. A sensação de vergonha acentuada prejudica a realização do exame, pois a mulher não consegue relaxar, o que torna o exame dolorido em decorrência de contrações na musculatura pélvica (BAIA EM, et al., 2018).

A não adesão do exame também está relacionada ao conhecimento insuficiente das mulheres idosas, segundo o estudo realizado por Feitosa LMH, et al. (2017), onde foi apontado que $75 \%$ das idosas tinham um conhecimento indevido em relação ao EGP, pois nas décadas de 50 e 60 as mulheres tinham menos referências sobre o CCU por não existiam atividades que levassem à promoção e prevenção de doenças. Já Sardinha AHL, et al. (2021), apresentaram evidências de que idosas com baixa escolaridade possuem um nível de instrução menor e chance maior para o desenvolvimento do CCU. 
De acordo com Batista AFC e Caldas CP (2017), a família tem um papel essencial na aceitação do plano de prevenção, pois o alargamento da expectativa de vida dos idosos gera também evoluções de doenças crônicas que podem causar incapacidade funcional. É no contexto familiar que se cria os cuidados e interações afetivas para o íntegro desenvolvimento da saúde da pessoa idosa que decorre de atitudes no cotidiano familiar e permite o reconhecimento de doenças, além de incentivar o autocuidado e a busca por um atendimento de saúde com a promoção do apoio emocional (BATISTA AFC e CALDAS CP, 2017).

Existem doutrinas e discriminações que emergem de uma visão antiga e que precisam de mudanças rotineiras, sendo esses fatores provenientes da própria visão da pessoa idosa, da família, sociedade e dos profissionais de saúde quanto ao envelhecimento (BATISTA AFC e CALDAS CP, 2017). Faz-se necessário, de acordo com Batista AFC e Caldas CP (2017), o entendimento e compreensão por parte da sociedade para com a pessoa idosa, visto que ela também precisa ser acompanhada, respeitada e tratada, tendo como foco o envelhecimento saudável e ativo, devendo ser considerados os hábitos de vida bem como suas atitudes frente à velhice.

Segundo Tavares DL, et al. (2018), os idosos vem cada dia mais apresentando uma vida sexual ativa e isso gera uma melhora na qualidade de vida, porém, existem tabus que são direcionados a eles dificultando a implementação de políticas públicas e fazendo com que se tornem expostos e vulneráveis a Infecções Sexualmente Transmissíveis (IST). Ainda no estudo de Tavares DL, et al. (2018), os autores dizem que a contaminação por Papiloma Vírus Humano (HPV) e outras IST podem vir a apresentar maiores incidências, uma vez que os idosos são tidos como seres assexuados e não são vistos como uma população de risco, não sendo, portanto, incentivados quanto à prática sexual segura.

O CCU está relacionado a infecções constantes de subtipos do vírus HPV, principalmente o HPV-16 e o HPV-18, que correspondem a 70\% dos cânceres cervicais (INCA, 2021). Na sua fase inicial, o CCU pode não apresentar sintomas, por ser uma patologia de evolução lenta mas, nos casos mais avançados, pode apresentar sangramento vaginal intermitente ou depois das relações sexuais, secreção vaginal irregular e dor abdominal relacionada às queixas urinárias ou intestinais (INCA, 2020). $O$ tratamento dependerá do avanço da doença, da dimensão do tumor e dos motivos pessoais das pacientes. Dentre os tratamentos para o CCU, é possível citar a eletrocirurgia (lesão precursora), a cirurgia, a quimioterapia e a radioterapia (INCA, 2021).

O vírus HPV surge através do contato direto com a pele ou com a mucosa contaminada, sendo sua forma mais prevalente a por meio sexual, que engloba contato oral-genital, genital-genital ou, da mesma forma, manual-genital. A contaminação pode ocorrer por barreira transplacentária, mesmo na ausência da penetração anal e vaginal (INCA, 2020). As mulheres são mais suscetíveis ao HPV por meio de fatores ligados à imunidade, à genética, início precoce da vida sexual, quantidade elevada de parceiros sexuais e gestações, uso de anticoncepcional, imunossupressão e idade (INCA, 2021; INCA, 2020).

Embora no Brasil a Atenção Básica (AB) seja um direito universal, a utilização dos serviços de saúde por parte dos idosos sofre influência da sua situação socioeconômica e da sua família. Idosos com renda mensal baixa denotam uma condição de saúde inferior se comparados aos idosos com situação socioeconômica superior, com menor adesão ao sistema de saúde. Os serviços de saúde e suas ações não se atentam às reais necessidades dos idosos, desconsideram a debilidade do serviço e não se atentam à promoção da autonomia apesar da legislação assegurar ao idoso uma assistência integral (BATISTA AFC e CALDAS CP, 2017).

Mesmo diante de uma ampliação da saúde, a pessoa idosa ainda é abordada de forma limitada a doenças crônicas, sendo atendida de forma isolada e sem prosseguimento, sendo desconsiderado o impacto na qualidade de vida(BATISTA AFC e CALDAS CP, 2017). A saúde das pessoas idosas não se limita apenas a inexistência de doença, devendo ser considerado também o seu nível de independência, por isso, deve ser feita uma avaliação multidimensional que considere tanto o bem-estar psicossocial quanto a precisão de ações da equipe multidisciplinar (TAVARES DL, et al., 2018).

O preconceito por parte dos profissionais de saúde frente a algumas situações ainda é encoberto e é comum depararmos com profissionais que não possuem conhecimento sobre o envelhecimento, atribuindo à idade determinadas manifestações patológicas, outros possuem conceitos antigos em relação ao CCU, e não 
utilizam a maneira correta para o rastreamento de idosas frente à prevenção e detecção precoce (BATISTA AFC e CALDAS CP, 2017). A percepção que os profissionais de saúde possuem em relação à pessoa idosa, segundo o estudo de Batista AFC e Caldas CP (2017), interfere no acompanhamento e no cuidado, visto que nossos hábitos cercam a forma de acolher.

De acordo com a Resolução Conselho Federal de Enfermagem (COFEN) № 385/2011, a coleta do Papanicolau é uma prática de cuidado, procedimento que exige conhecimento científico, sendo o profissional enfermeiro capacitado para realização do exame, atentando para a capacitação contínua necessária (COFEN, 2021). Durante a consulta, o profissional de saúde deve orientar as pacientes, uma vez que, no ato do exame ele é responsável por fornecer informações à mulher, realizar o acolhimento e manter a privacidade na consulta de enfermagem. Nesse sentido, o profissional deve desenvolver estratégias de como abordar e realizar o acolhimento, pois o nível de satisfação dessas idosas influenciam para um possível retorno ou não (ACOSTA DF, et al., 2017).

Diante dos fatores impeditivos para a realização do EGP, percebe-se a necessidade do enfermeiro em trabalhar ações educativas de promoção da saúde. É importante salientar que os profissionais de saúde busquem formas para reduzir o sentimento de vergonha no decorrer da realização do exame, agindo de forma empática, de modo que a idosa se sinta mais confortável, uma vez que, caso essa mulher se sinta constrangida a mesma pode desistir de realizar o exame e essa desistência pode colocar em risco a sua saúde. Cabe ao profissional realizar um atendimento humanizado, esclarecer os benefícios de um acompanhamento regular e da detecção precoce de anomalias, e explicar o procedimento que será realizado (OLIVEIRA DS, et al., 2019).

Andrade MI, et al. (2021), ressaltam o dever do profissional de saúde quanto mentor, influenciando nas condutas e atitudes dessa população, sobretudo em relação ao EGP e outras práticas que abarcam a saúde da mulher. Segundo Silva JP, et al. (2018), o profissional de enfermagem está ligado à comunidade de modo contínuo, tendo maior contato com a população no nível de Atenção Básica $(A B)$, de modo que a educação em saúde é essencial na AB. Silva JP, et al. (2018), reforçam também que a Estratégia Saúde da Família (ESF) promova a educação continuada, com informações sobre a importância do exame, de forma a quebrar barreiras da não realização do EGP.

Acosta DF, et al. (2017), ressaltam que idosas enfrentam mais obstáculos para a realização do exame preventivo, de modo que há a necessidade de a equipe de enfermagem promover ações educativas e participativas, devendo esclarecer todas as dúvidas em relação ao EGP e sua importância para a prevenção do CCU junto à essa população. Já Andrade MI, et al. (2019), salientam que a prevenção do CCU em idosas inicia-se mediante informações, e que, ao serem informadas sobre o CCU, essas idosas entendem e se tornam adeptas a realização das medidas preventivas, sobretudo, quando estabelecido um vínculo de confiança com as mesmas.

Oliveira DS, et al. (2019), enfatizam que a equipe de enfermagem deve fazer a busca ativa das idosas, visto que, essa população está comumente exposta ao risco de desenvolverem o CCU. A equipe de enfermagem deve promover campanhas e esclarecimentos, incentivar a realização do exame juntamente com os Agentes Comunitários de Saúde (ACS), propagando informações para a população idosa (OLIVEIRA DS, et al., 2019). Segundo Maciel NS, et al. (2021), diante da busca ativa, podem ser empregadas também ações educativas na sociedade, visitas domiciliares as idosas que estão com o exame atrasado ou que não executaram para conhecer essa população e estabelecer vínculo de confiança de forma humanística, visando o bem-estar.

Moura JBLC e Silva GV (2017), destacam que na AB e a ESF o enfermeiro atua como membro da equipe multiprofissional, é responsável por monitorar a população idosa, junto com o ACS, que, por sua vez, tem papel primordial em realizar visita nas residências, orientar sobre a importância da coleta do Papanicolau, e convidá-la a procurar a UBS do seu território. Medeiros ATN, et al. (2021), detalham que o enfermeiro atua na organização da agenda, realiza consulta de enfermagem, coleta o material citopatológico e a educação em saúde, orienta como deve ser realizado o exame EGP para que esse cuidado aconteça de forma efetiva e com qualidade. 
A educação em saúde contribui para a busca de soluções e construção crítica das pessoas em relação à saúde, pois promove um papel fundamental para que as pessoas se mantenham saudáveis, considerando sua realidade e proporciona conhecimentos para a vida diária, com grande influência sobre a vida dos usuários, ao tempo que oferece assistência para aceitação de novas práticas e comportamento de saúde (BATISTA AFC e CALDAS CP, 2017).

\section{CONSIDERAÇÕES FINAIS}

O presente estudo observou que a não adesão ao exame ginecológico Papanicolau ainda é um problema de saúde pública, pois envolve barreiras para sua realização. Essas barreiras são a falta de conhecimento, a baixa escolaridade, o preconceito, os fatores culturais, o medo, a vergonha e o constrangimento da exposição da genitália ao profissional. O enfermeiro tem um papel fundamental no esclarecimento de como ocorre a coleta citopatológica, por meio da disseminação de informações para mulheres de todas as idades. Visando o bem-estar das mulheres acompanhadas, devem ser planejadas ações educativas com atendimento humanizado, além da realização de visitas domiciliares para conhecimento desta população.

\section{REFERÊNCIAS}

1. ACOSTA DF, et al. Vivenciando o exame Papanicolau: entre o (não) querer e o fazer. Revista de Enfermagem da Universidade Federal de Pernambuco (UFPE), 2017; 11(8): 3031-3038.

2. ANDRADE MI, et al. Fatores que dificultam a adesão de mulheres ao exame Papanicolau. Revista Journal of Development Research, 2021; 11(06): 48023-48026.

3. BAIA EM, et al. Dificuldades enfrentadas pelas mulheres para realizar o exame Papanicolau: revisão integrativa. Revista Nursing (São Paulo), 2018; 21(238): 2068-2074.

4. BARRETO AMMA, et al. Intervenção educativa em saúde para idosas à cerca do exame Papanicolau. Revista de Pesquisa Cuidado é Fundamental Online, 2018; 10(Esp.):252-254.

5. BATISTA AFC, CALDAS CP. Fatores que interferem na adesão da mulher idosa a programas de prevenção do câncer ginecológico. Revista de Enfermagem da Universidade do Estado do Rio de Janeiro (UERJ), 2017; 25: 21.839 p.4.

6. CAMPOS EA, et al. "Uma doença da mulher": experiência e significado do câncer cervical para mulheres que realizaram o Papanicolau. Revista Interface-Comunicação, Saúde, Educação, 2017; 21(61); 385-396.

7. CARNEIRO C, et al. Fatores que influenciam na realização do exame Papanicolaou: revisão integrativa da literatura. Repositório Institucional UniGuairaca, 2020; p.10.

8. DIAS EG, et al. Importância atribuída pelas mulheres à realização do exame Papanicolaou. Saúde em Redes, 2017; 3(4): 350-357.

9. CONSELHO FEDERAL DE ENFERMAGEM (COFEN). Resolução $n^{\circ}=385 / 2011$. Normatiza a execução pelo enfermeiro da coleta de material para coleta da citologia oncótica pelo método de papanicolau.

10. FEITOSA LMH, et al. Realização do colpocitológico em idosas. Revista de Enfermagem da Universidade Federal de Pernambuco (UFPE), 2017; 11(9):3321-9.

11. IGLESIAS GA, et al. Conhecimento e adesão ao Papanicolau de mulheres de uma rede de Atenção Primária à Saúde. Revista de Ciências Médicas, 2019; 28(1):21-30 p.23

12. INSTITUTO NACIONAL DO CÂNCER (INCA), 2019. Controle do câncer do colo de útero, conceito e magnitude. Disponível em: https://www.inca.gov.br/controle-do-cancer-do-colo-do-utero/conceito-e-magnitude. Acessado em: 7 de setembro de 2021.

13. INSTITUTO NACIONAL DE CÂNCER (INCA), 2021. Câncer de Colo de Útero Disponível em: https://www.inca.gov.br/tipos-de-cancer/cancer-do-colo-do-utero. Acessado em: 11 de novembro de 2021.

14. INSTITUTO NACIONAL DE CÂNCER (INCA), 2021. Detecção precoce. Disponível em: https://www.inca.gov.br/en/node/1194. Acessado em: 11 de novembro de 2021.

15. INSTITUTO NACIONAL DE CÂNCER (INCA), 2020. Fatores que deixam as mulheres mais suscetíveis a contraírem o HPV. Disponível em: https://www.inca.gov.br/perguntas-frequentes/alem-infeccao-pelo-hpv-ha-outros-fatores-queaumentam-o-risco-uma-mulher. Acessado em: 11 de novembro de 2021.

16. INSTITUTO NACIONAL DO CÂNCER (INCA), 2021. Prevenção do câncer do colo do útero. Disponível em: https://www.inca.gov.br/controle-do-cancer-do-colo-do-utero/acoes-de-controle/prevencao. Acessado em: 5 de setembro de 2021.

17. INSTITUTO NACIONAL DE CÂNCER (INCA), 2020. Relação entre HPV e o Câncer. Disponível em: https://www.inca.gov.br/perguntas-frequentes/hpv. Acessado em: 21 de novembro de 2021. 
18. LEITE BO, et al. A Percepção das Mulheres Idosas Sobre o Exame de Prevenção de Câncer do Colo de Útero. Revista de Pesquisa da Universidade Federal do Rio Janeiro, 2019; 11(5):1347.

19. LIMA MB, et al. Motivos que influenciam a não realização do exame Papanicolau segundo a percepção de mulheres. Revista Temas em Saúde, 2017; 17(1): 353-369.

20. LIMA WF, et al. Perfil dos exames de Citopatologia ginecológica realizados em uma clínica do norte do Piauí: análise documental. Revista Research, Society And Development, 2020; 9(7): 3259/74440.

21. MACIEL NS, et al. Busca ativa para o aumento da adesão ao Exame Papanicolau. Revista de Enfermagem da Universidade Federal de Pernambuco (UFPE), 2021; 15 e 247678 p.8.

22. MEDEIROS ATN, et al. Ações do enfermeiro na prevenção do câncer do colo do útero na Atenção Básica. Revista Research, Society And Development, 2021; 10(10): 348101018519.

23. MINISTÉRIO DA SAÚDE (MS). Citologia em meio líquido para rastreamento de câncer de colo de útero e lesões precursoras. 2019. Disponível em:http://conitec.gov.br/images/Relatorios/2019/Relatrio-Citologia-em-MeioLquido_FINAL_497_2019.pdf. Acessado em: 28 de setembro de 2021.

24. MOREIRA AS, ANDRADE EGS. A importância do exame papanicolau na saúde da mulher. Revista de Iniciação Científica e Extensão, 2018; 1(3): 267-71.

25. MOURA JBLC, DA SILVA GV. Papanicolaou: Refletindo sobre o cuidado de Enfermagem na Atenção Básica. Revista Pró-UniverSUS, 2017; 8(1) 12:16.

26. OLIVEIRA DS, et al. Atuação da enfermeira frente aos fatores que interferem na adesão de mulheres idosas ao exame de Papanicolau. Revista Enfermagem Contemporânea, 2019; 8(1): 87-93.

27. SANTOS $A D$, et al. Concepção de mulheres idosas sobre a sexualidade na velhice. Revista de Enfermagem da Universidade Federal de Pernambuco (UFPE), 2019; 13:241752.

28. SARDINHA AHL, et al. Associação entre variáveis demográficas e estadiamento de câncer cervical em idosas: estudo retrospectivo. Revista Online Brazilian Journal Of Nursing, 2021; 20: e 20216479.

29. SECRETARIA ESTADUAL DE SAÚDE DE PERNAMBUCO. Cartilha de atenção integral à saúde da mulher. 2018. Disponível em: http://portal.saude.pe.gov.br/sites/portal.saude.pe.gov.br/files/cartilha_a5_saudedamulher_2018.pdf. Acessado em: 3 de setembro de 2021.

30. SELBAC MT, et al. Mudanças comportamentais e fisiológicas determinadas pelo ciclo biológico feminino - climatério a menopausa. Rev. Aletheia, 2018; 51(1-2), 177-190.

31. SILVA JP, et al. Exame Papanicolau: fatores que influenciam a não realização do exame em mulheres de 40 a 65 anos. Revista Archives of Health Sciences (Online), 2018; 25(2)15-19.

32. SILVA MO, et al. Fatores relacionados à não adesão ao exame citopatológico em mulheres na melhor idade: uma revisão sistemática com Metassíntese. Revista Brazilian Journal of Development (BJD), 2020; 6(8); 60925-60934.

33. SIMÕES CS, et al. Diagnóstico laboratorial das lesões precursoras do câncer de colo do útero: Revisão sistemática. Revista Brazilian Journal of Health Review, 2021; 4(4): 15534-15558.

34. TAVAREZ DL, et al. Prevalência do exame citopatológico cérvico-vaginal e microflora em idosas de Santa Maria, Rio Grande do Sul. Revista Saúde (Santa Maria), 2018; 44(1): I-8. 